\title{
The Log-concavity Property Associated to Hyperjacobsthal and Hyperjacobsthal-Lucas Sequences
}

\author{
Moussa Ahmia ${ }^{1, *}$, Hacène Belbachir ${ }^{2}$ \\ ${ }^{1}$ Department of Mathematic, University of Mohamed Seddik Ben yahia, Jijel, Algeria \\ ${ }^{2}$ Faculty of Mathematics, University of Sciences and Technology Houari Boumediene, Algiers, Algeria \\ *Corresponding author: ahmiamoussa@email.com
}

Received January 20, 2018; Revised March 26, 2018; Accepted June 25, 2018

\begin{abstract}
In this paper, we show the log-concavity properties for the hyperjacobsthal, hyperjacobsthal-Lucas and associated sequences. Further, we investigate the $q$-log-concavity property.
\end{abstract}

Keywords: Hyperjacobsthal numbers, hyperjacobsthal-Lucas numbers, log-concavity, q-log-concavity

Cite This Article: Moussa Ahmia, and Hacène Belbachir, "The Log-concavity Property Associated to Hyperjacobsthal and Hyperjacobsthal-Lucas Sequences." Turkish Journal of Analysis and Number Theory, vol. 6, no. 3 (2018): 107-110. doi: 10.12691/tjant-6-3-8.

\section{Introduction}

Let $\left(x_{n}\right)_{n}$ be a sequence of nonnegative numbers. If for all $>0, x_{j}^{2} \geq x_{j-1} x_{j+1}$ (respectively $\left.x_{j}^{2} \leq x_{j-1} x_{j+1}\right)$, the sequence is called log-concave (respectively log-convex), which is equivalent to $x_{i} x_{j} \geq x_{i-1} x_{j+1}$ (respectively $\left.x_{i} x_{j} \geq x_{i-1} x_{j+1}\right)$ for $j \geq i \geq 1$.

The log-concave and log-convex sequences arise often in combinatorics, algebra, geometry, analysis, probability and statistics and have been extensively investigated. We refer the reader to $[1,2,3]$ for log-concavity.

Let $\left(f_{n}(q)\right)_{n \geq 0}$ be a sequences of polynomials in $q$. If for each $n \geq 1, f_{n}^{2}(q)-f_{n-1}(q) f_{n+1}(q)$ has nonnegative coefficients as a polynomials in $q$; we say that $\left(f_{n}(q)\right)_{n \geq 0}$ is $q$-log-concave. The $q$-log-concavity of polynomials have been extensively studied; see for instance $[4,5,6]$.

In $[7,8]$, some properties of hyperfibonacci numbers $F_{n}^{[r]}$ and hyperlucas numbers $L_{n}^{[r]}$ are given. For Fibonacci numbers $F_{n}$ and Lucas numbers $L_{n}$, it is well known that $\left(F_{2 n+1}\right)$ and $\left(L_{2 n}\right)$ are log-concave (see [9]).

L. Zheng and R. Liu [10] gived some properties of the hyperfibonacci numbers and hyperhucas numbers, and investigated the log-concavity and log-convexity of these numbers. Finaly, they also studied the log-concavity (logconvexity) of generalized hyperfibonacci numbers and hyperlucas numbers. In [11], we established these properties for hyperpell numbers and hyperpell-Lucas numbers.

In section two, we give the definitions and some properties of hyperjacobsthal and hyperjacobsthal-Lucas sequences. In section three, we establish the generating functions of these sequences. In section 4, we discuss their $\log$ - concavity. In addition, we investigate the $q$-log-concavity of some polynomials related to hyperjacobsthal and hyperjacobsthal-Lucas numbers.

\section{Definitions}

Dil and Mezö [8] introduced the hyperfibonacci numbers $F_{n}^{[r]}$ and hyperlucas numbers $L_{n}^{[r]}$ to be

$$
\begin{aligned}
& F_{n}^{[r]}=\sum_{k=0}^{n} F_{k}^{[r-1]}, \text { with } F_{n}^{[0]}=F_{n}, \\
& L_{n}^{[r]}=\sum_{k=0}^{n} L_{k}^{[r-1]}, \text { with } L_{n}^{[0]}=L_{n},
\end{aligned}
$$

where $\mathrm{r}$ is a positive integer, and $F_{n}$ and $L_{n}$ are Fibonacci and Lucas numbers, respectively.

Definition 2.1. Let $r$ be positive integer. The hyperjacobsthal numbers $J_{n}^{[r]}$ and hyperjacobsthal-Lucas numbers $j_{n}^{[r]}$ are defined as follows

$$
J_{n}^{[r]}=\sum_{k=0}^{n} J_{k}^{[r-1]}, j_{n}^{[r]}=\sum_{k=0}^{n} j_{k}^{[r-1]} .
$$

where $J_{n}^{[0]}=J_{n}, \quad j_{n}^{[0]}=j_{n}$, and $\left(J_{n}\right)_{n}$ and $\left(j_{n}\right)_{n}$ are Jacobsthal and Jacobsthal-Lucas sequences, respectively.

The initial values of $\left(J_{n}^{[r]}\right)_{n}$ and $\left(j_{n}^{[r]}\right)_{n}$ are as follows

\begin{tabular}{|c|c|c|c|c|c|c|c|c|}
\hline$n$ & 0 & 1 & 2 & 3 & 4 & 5 & 6 & 7 \\
\hline$J_{n}^{[1]}$ & 0 & 1 & 2 & 5 & 10 & 21 & 42 & 85 \\
\hline$j_{n}^{[1]}$ & 2 & 3 & 8 & 15 & 32 & 63 & 128 & 255 \\
\hline
\end{tabular}

Now we recall some formulas for Jacobsthal and Jacobsthal-Lucas numbers. It is well know that the Binet forms of $J_{n}$ and $j_{n}$ are

$$
J_{n}=\frac{2^{n}-(-1)^{n}}{3}, j_{n}=2^{n}+(-1)^{n} .
$$


See for instance [12].

The sequences $\left(J_{n}\right)_{n}$ and $\left(j_{n}\right)_{n}$ satisfy the following recurrences

$$
W_{n}=W_{n-1}+2 W_{n-2}, n \geq 2 .
$$

For more details, see for instance [13].

It follows from (2.1) that the following formulas hold:

$$
\begin{gathered}
J_{n+2}^{2}-J_{n+1} J_{n+3}=(-1)^{n+1} 2^{n+1}, \\
j_{n+2}^{2}-j_{n+1} j_{n+3}=9(-1)^{n} 2^{n+1} .
\end{gathered}
$$

It is easy to see, for example by induction, that

$$
\begin{gathered}
J_{n+1}=2 J_{n}+(-1)^{n}, j_{n+1}=2 j_{n}-3(-1)^{n} . \\
J_{n} \geq n-1 \text { and } j_{n} \geq n \text { for } n \geq 1 .
\end{gathered}
$$

The generating function of Jacobsthall numbers and Jacobsthal-Lucas numbers, denoted $G_{J}(t)$ and $G_{j}(t)$, are respectively

$$
G_{J}(t):=\sum_{n=0}^{+\infty} J_{n} t^{n}=\frac{t}{1-t-2 t^{2}}
$$

and

$$
G_{j}(t):=\sum_{n=0}^{+\infty} j_{n} t^{n}=\frac{2-2 t}{1-t-2 t^{2}}
$$

So, we establish the generating function of hyperjacobsthal and hyperjacobsthal-Lucas numbers using respectively

$$
J_{n}^{[r]}=J_{n-1}^{[r]}+J_{n}^{[r-1]} \text { and } j_{n}^{[r]}=j_{n-1}^{[r]}+j_{n}^{[r-1]} .
$$

The generating functions of hyperjacobsthal numbers and hyperjacobsthal-Lucas numbers are

$$
G_{J}^{[r]}(t):=\sum_{n=0}^{+\infty} J_{n}^{[r]} t^{n}=\frac{t}{\left(1-t-2 t^{2}\right)(1-t)^{r}},
$$

and

$$
G_{j}^{[r]}(t):=\sum_{n=0}^{+\infty} j_{n}^{[r]} t^{n}=\frac{2-2 t}{\left(1-t-2 t^{2}\right)(1-t)^{r}} .
$$

\section{The Log-concavity Property}

We start the section by some useful lemmas.

Lemma 3.1. [15] If the sequences $\left(x_{n}\right)_{n}$ and $\left(y_{n}\right)_{n}$ are log-concave, then so is their ordinary convolution $z_{n}=\sum_{k=0}^{n} x_{k} y_{n-k}, n=0,1,2 \ldots$.

Lemma 3.2. [15] If the sequences $\left(x_{n}\right)_{n}$ is log-concave, then so is the binomial convolution $z_{n}=\sum_{k=0}^{n}\left(\begin{array}{l}n \\ k\end{array}\right) x_{k}$, $n=0,1,2 \ldots$.

The following result deals with the log-concavity of hyperjacobsthal and hyperjacobsthal-Lucas sequences.

Theorem 3.3. The sequences $\left(J_{n}^{[r]}\right)_{n \geq 0}$ and $\left(j_{n}^{[r]}\right)_{n \geq 0}$ are log-concave for $r \geq 2$ and $r \geq 3$ respectively.

Proof. To prove the results, we use the following relations

$$
\mathrm{J}_{\mathrm{n}}^{[2]}=\frac{1}{4}\left(\mathrm{~J}_{\mathrm{n}+4}-2 \mathrm{n}-5\right) \text { and } \mathrm{j}_{\mathrm{n}}^{[2]}=\frac{1}{4}\left(\mathrm{j}_{\mathrm{n}+4}-2 \mathrm{n}-9\right)(3.1)
$$

When $\mathrm{n}=1,\left(\mathrm{~J}_{\mathrm{n}}^{[2]}\right)^{2}-\mathrm{J}_{\mathrm{n}-1}^{[2]} \mathrm{J}_{\mathrm{n}+1}^{[2]}=1>0$. When $\mathrm{n} \geq 2$ it follows from (2.2), (2.4), (2.5) and (3.1)

$$
\begin{aligned}
& \left(J_{n}^{[2]}\right)^{2}-J_{n-1}^{[2]} J_{n+1}^{[2]} \\
& =\frac{1}{16}\left(\left[J_{n+4}-(2 n+5)\right]^{2}\right. \\
& -\left[J_{n+3}-(2 n+3)\right] \times\left[J_{n+5}-(2 n+7)\right] \\
& =\frac{1}{4}\left[(-1)^{n+1} 2^{n+1}+(2 n-1) J_{n+1}+2(-1)^{n+1}+1\right] \\
& \geq \frac{1}{4}\left[(-1)^{n+1} 2^{n+1}+(2 n-1) \times \frac{2^{n}-(-1)^{n}}{3}-1\right] .
\end{aligned}
$$

There exist two cases. If $\mathrm{n}$ is even, then

$$
\left(J_{n}^{[2]}\right)^{2}-J_{n-1}^{[2]} J_{n+1}^{[2]}=\frac{2 n-4}{12}\left(2^{n+1}+1\right)>0,
$$

else

$$
\left(J_{n}^{[2]}\right)^{2}-J_{n-1}^{[2]} J_{n+1}^{[2]}=\frac{2 n+2}{12}\left(2^{n+1}-1\right)>0 .
$$

Then $\left(J_{n}^{[2]}\right)_{n \geq 0}$ is log-concave. By induction hypothesis and Lemma 3.1 the sequence $\left(J_{n}^{[r]}\right)_{n \geq 0}(r \geq 2)$ is log-concave.

One can verify that

$$
j_{n}^{[3]}=\frac{1}{8}\left[j_{n+6}-2(n+1)(n+9)+31\right] .
$$

It follows from (2.3), (2.5) and (3.3) that

$$
\begin{aligned}
& \left(\mathrm{j}_{\mathrm{n}}^{[3]}\right)^{2}-\mathrm{j}_{\mathrm{n}-1}^{[3]} \mathrm{j}_{\mathrm{n}+1}^{[3]} \\
& =\frac{1}{16}\left(\left(\mathrm{j}_{\mathrm{n}+6}-2(\mathrm{n}+1)(\mathrm{n}+9)+31\right)^{2}\right. \\
& -\left(\mathrm{j}_{\mathrm{n}+5}-2 \mathrm{n}(\mathrm{n}+8)+31\right) \\
& \left.\times\left(\mathrm{j}_{\mathrm{n}+6}-2(\mathrm{n}+2)(\mathrm{n}+10)+31\right)\right) \\
& =\frac{1}{16}\left[-9(-1)^{\mathrm{n}+3} 2^{\mathrm{n}+3}+(2 \mathrm{n}(2 \mathrm{n}+4)-1) \mathrm{j}_{\mathrm{n}+3}\right. \\
& \left.+3(4 \mathrm{n}+17)(-1)^{\mathrm{n}+3}+\left(2 \mathrm{n}^{2}+20 \mathrm{n}+5\right)\right] .
\end{aligned}
$$

For $\mathrm{n} \geq 1$, there exist two cases. If $\mathrm{n}$ is even, we get

$$
\left(\mathrm{j}_{\mathrm{n}}^{[3]}\right)^{2}-\mathrm{j}_{\mathrm{n}-1}^{[3]} \mathrm{j}_{\mathrm{n}+1}^{[3]}=\frac{1}{16}\left[8 \times 2^{\mathrm{n}+3}+2 \mathrm{n}(\mathrm{n}+4) 2^{\mathrm{n}+3}\right], \text { ? }
$$

else

$$
\begin{aligned}
& \left(\mathrm{j}_{\mathrm{n}}^{[3]}\right)^{2}-\mathrm{j}_{\mathrm{n}-1}^{[3]} \mathrm{j}_{\mathrm{n}+1}^{[3]} \\
& =\frac{1}{16}\left[\left(2 \mathrm{n}^{2}+8 \mathrm{n}-10\right) 2^{\mathrm{n}+3}+\left(4 \mathrm{n}^{2}+40 \mathrm{n}+100\right)\right] .
\end{aligned}
$$

Hence $\left(\mathrm{j}_{\mathrm{n}}^{[3]}\right)_{\mathrm{n} \geq 0}$ is log-concave. By induction hypothesis and Lemma 3.1 the sequence $\left(\mathrm{j}_{\mathrm{n}}^{[\mathrm{r}]}\right)_{\mathrm{n} \geq 0}(\mathrm{r} \geq 3)$ is $\log$ concave. This completes the proof of Theorem 3.3. 
Then we have the following corollary.

Corollary 3.4. The sequences $\left(\sum_{\mathrm{k}=0}^{\mathrm{n}}\left(\begin{array}{l}\mathrm{n} \\ \mathrm{k}\end{array}\right) \mathrm{J}_{\mathrm{k}}^{[\mathrm{r}]}\right)_{\mathrm{n} \geq 0}$ and $\left(\sum_{\mathrm{k}=0}^{\mathrm{n}}\left(\begin{array}{l}\mathrm{n} \\ \mathrm{k}\end{array}\right) \mathrm{j}_{\mathrm{k}}^{[\mathrm{r}]}\right)_{\mathrm{n} \geq 0}$ are $\log$-concave for $\mathrm{r} \geq 2$ and $\mathrm{r} \geq 3$ respectively.

Proof. By Lemma 3.2.

Now we establish the log-concavity of order two of the sequences $\left(J_{n}^{[r]}\right)_{n \geq 0}$ and $\left(j_{n}^{[r]}\right)_{n \geq 0}$ for some special sub-sequences.

Theorem 3.5. Let $\mathrm{n} \geq 1$

$$
T_{n}:=\left(\mathrm{J}_{\mathrm{n}}^{[2]}\right)^{2}-\mathrm{J}_{\mathrm{n}-1}^{[2]} \mathrm{J}_{\mathrm{n}+1}^{[2]} \text { and } R_{n}:=\left(\mathrm{g}_{\mathrm{n}}^{[3]}\right)^{2}-\mathrm{j}_{\mathrm{n}-1}^{[3]} \mathrm{j}_{\mathrm{n}+1}^{[3]} \text {. }
$$

Then, the sub-sequences $\left(T_{2 n}\right)_{n \geq 1},\left(T_{2 n+1}\right)_{n \geq 0},\left(R_{2 n}\right)_{n \geq 1}$ and $\left(R_{2 n+1}\right)_{n \geq 0}$ are log-concave.

Proof. From (2.4), we get

$$
\begin{gathered}
J_{2 n+1}^{2}-J_{2 n-1} J_{2 n+3}=-2^{2 n-1}, n \geq 1 . \\
J_{2 n+2}^{2}-J_{2 n} J_{2 n+4}=2^{2 n}, n \geq 1 . \\
j_{2 n+3}^{2}-j_{2 n+1} j_{2 n+5}=9 \times 2^{2 n+1}, n \geq 1 .
\end{gathered}
$$

It follows form (3.2) and (3.5) that

$$
\begin{aligned}
& T_{2 n}^{2}-T_{2(n-1)} T_{2(n+1)} \\
& =\frac{1}{16}\left[-2^{2 n+1}+(4 n-1) J_{2 n+1}+3\right]^{2} \\
& -\left[-2^{2 n-1}+(4 n-5) J_{2 n-1}+3\right] \\
& \times\left[-2^{2 n-1}+(4 n+3) J_{2 n+3}+3\right] \\
& =2^{2 n-1}\left[\frac{7}{9} 2^{2 n-1}-\frac{10}{9}\right]+\frac{1}{9}>0 .
\end{aligned}
$$

Then $\left(T_{2 n}\right)_{n \geq 0}$ is log-concave.

It follows from (3.2) and (3.6)

$$
\begin{aligned}
& T_{2 n+1}^{2}-T_{2 n-1} T_{2 n+3} \\
& =\frac{1}{16}\left[2^{2 n+2}+(4 n+1) J_{2 n+2}-1\right]^{2} \\
& -\left[2^{2 n}+(4 n-3) J_{2 n-1}-1\right] \\
& \times\left[-2^{2 n+4}+(4 n+5) J_{2 n+4}-1\right] \\
& =\frac{1}{16}\left[(4 n+1)^{2} 2^{2 n}+16 J_{2 n} J_{2 n+4}\right. \\
& \left.+(2 n-8) 2^{2 n}+(12 n+23)\left(2^{2 n}+2\right)\right] .
\end{aligned}
$$

Then $\left(T_{2 n+1}\right)_{n \geq 0}$ is log-concave.

Similarly, by applying (3.3) and (3.7), we have

$$
\begin{aligned}
& R_{2 n}^{2}-R_{2(n-1)} R_{2(n+1)} \\
& =\frac{2^{4 n+6}}{256}\left[\left(8 n^{2}+16 n+8\right)^{2}-64 n^{2}\left(4 n^{2}+4 n+4\right)^{2}\right] \\
& =2^{4 n+2}\left[2 n^{2}+4 n+1\right]>0 .
\end{aligned}
$$

Then $\left(R_{2 n}\right)_{n \geq 1}$ is log-concave.

By same technic, we obtain

$$
R_{2 n+1}^{2}-R_{2 n-1} R_{2 n+3}>0 .
$$

Then $\left(R_{2 n+1}\right)_{n \geq 0}$ is log-concave. This completes the proof.

Then we have the following corollaries.

Corollary 3.6. The sequences $\left(\sum_{\mathrm{k}=0}^{\mathrm{n}}\left(\begin{array}{l}\mathrm{n} \\ \mathrm{k}\end{array}\right) \mathrm{T}_{2 \mathrm{k}}^{[\mathrm{r}]}\right)_{\mathrm{n} \geq 1}$ and $\left(\sum_{\mathrm{k}=0}^{\mathrm{n}}\left(\begin{array}{l}\mathrm{n} \\ \mathrm{k}\end{array}\right) \mathrm{T}_{2 \mathrm{k}+1}^{[\mathrm{r}]}\right)_{\mathrm{n} \geq 0}$ are log-concave.

Proof. By Lemma 3.2.

Corollary 3.7. The sequences $\left(\sum_{\mathrm{k}=0}^{\mathrm{n}}\left(\begin{array}{l}\mathrm{n} \\ \mathrm{k}\end{array}\right) \mathrm{R}_{2 \mathrm{k}}^{[\mathrm{r}]}\right)_{\mathrm{n} \geq 1}$ and $\left(\sum_{\mathrm{k}=0}^{\mathrm{n}}\left(\begin{array}{l}\mathrm{n} \\ \mathrm{k}\end{array}\right) \mathrm{R}_{2 \mathrm{k}+1}^{[\mathrm{r}]}\right)_{\mathrm{n} \geq 0}$ are log-concave.

Proof. By Lemma 3.2.

Now, we establish the $q$-log-concavity property as follows.

Theorem 3.8. Define, for $r \geq 1$, the polynomials

$$
J_{n, r}(q)=\sum_{k=0}^{n} \mathrm{~J}_{\mathrm{k}}^{[\mathrm{r}]} q^{k}, \quad j_{n, r}(q)=\sum_{k=0}^{n} \mathrm{j}_{\mathrm{k}}^{[\mathrm{r}]} q^{k} .
$$

The polynomials $J_{n, r}(q)$ and $j_{n, r}(q)$ are $q$-log-concave for $(r \geq 2)$ and $(r \geq 3)$ respectively.

Proof. When $\geq 1, r \geq 2$,

$$
\begin{aligned}
& {\left[J_{n, r}(q)\right]^{2}-J_{n-1, r}(q) J_{n+1, r}(q)} \\
& =\left[\sum_{k=0}^{n} \mathrm{~J}_{\mathrm{k}}^{[\mathrm{r}]} q^{k}\right]^{2} ? \sum_{k=0}^{n-1} \mathrm{~J}_{\mathrm{k}}^{[\mathrm{r}]} q^{k} \times \sum_{k=0}^{n+1} \mathrm{~J}_{\mathrm{k}}^{[\mathrm{r}]} q^{k} \\
& =\sum_{k=1}^{n}\left[\mathrm{~J}_{\mathrm{k}}^{[\mathrm{r}]} \mathrm{J}_{\mathrm{n}}^{[\mathrm{r}]}-\mathrm{J}_{\mathrm{k}-1}^{[\mathrm{r}]} \mathrm{J}_{\mathrm{n}+1}^{[\mathrm{r}]}\right] q^{k+n} .
\end{aligned}
$$

When $\geq 1, r \geq 3$, through computation, we get

$$
\begin{aligned}
& {\left[j_{n, r}(q)\right]^{2}-j_{n-1, r}(q) j_{n+1, r}(q)} \\
& =\mathrm{J}_{\mathrm{n}}^{[\mathrm{r}]} q^{n}+\sum_{k=1}^{n}\left[\mathrm{j}_{\mathrm{k}}^{[\mathrm{r}]} \mathrm{J}_{\mathrm{n}}^{[\mathrm{r}]}-\mathrm{j}_{\mathrm{k}-1}^{[\mathrm{r}]} \mathrm{j}_{\mathrm{n}+1}^{[\mathrm{r}]}\right] q^{k+n} .
\end{aligned}
$$

As $\left(J_{n}^{[r]}\right)_{n \geq 0}(r \geq 2)$ and $\left(j_{n}^{[r]}\right)_{n \geq 0}(r \geq 3)$ are log-concave, then the polynomials $J_{n, r}(q)$ and $j_{n, r}(q)$ are $q$-log-concave for $(r \geq 2)$ and $(r \geq 3)$ respectively.

\section{Concluding remarks}

We have discussed the log-concavity of hyperjacobsthal numbers and hyperjacobsthal-Lucas numbers. In addition, we estabilished the $q$-log-concavity of some polynomials related to the both numbers.

\section{Acknowledgements}

We would like to thank the referee for useful suggestions and several comments witch involve the quality of the paper. 


\section{References}

[1] F. Brenti, Log-concave and unimodal sequence in algebra, combinatorics and geometry: an update. Elec. Contemp. Math. 178 (1994, 1997), 71-84.

[2] R. P. Stanley, Log-concave and unimodal sequences in algebra, combinatorics, and geometry, Ann. New York Acad. Sci. 576 (1989), 500-534

[3] Y. Wang, Y.-N. Yeh, Log-concavity and LC-positivity, J. Combin. Theory Ser. A, 114 (2007), 195-210.

[4] L. M. Butler, The q-log concavity of q-binomial coefficients, J. Combin. Theory Ser. A 54 (1990), 54-63.

[5] W. Y. C. Chen, L. X. W. Wang and A. L. B. Yang, Schur positivity and the q-log-convexity of the Narayana polynomials, J. Algebr. Comb. 32 (2010), 303-338.

[6] B.-X. Zhu, Log-convexity and strong q-log-convexity for some triangular arrays, Adv. in. Appl. Math. 50(4) (2013), 595-606.

[7] N-N. Cao, F-Z. Zhao, Some Properties of Hyperfibonacci and Hyperlucas Numbers, Journal of Integer Sequences, 13(8) (2010), Article 10.8.8.

[8] A. Dil, I. Mezö, A symmetric algorithm for hyperharmonic and Fibonacci numbers,Appl. Math. Comput. 206 (2008), 942-951.

[9] N. J. A. Sloane, On-line Encyclopedia of Integer Sequences, http://oeis.org, (2014).
[10] L.-N. Zheng, R. Liu, On the Log-Concavity of the Hyperfibonacci Numbers and the Hyperlucas Numbers, J. Integer Sequences, Vol. 17 (2014), Article 14.1.4.

[11] M. Ahmia, H. Belbachir, A. Belkhir, The log-concavity and logconvexity properties associated to hyperpell numbers and hyperpell-lucas numbers, Annales Mathematicae et Informaticae. 43 (2014), 3-12.

[12] A. F. Horadam. Jacobsthal Representation Numbers. Fibonacci Quarterly, 34 (1) (1996), 40-54.

[13] A. F. Horadam. Jacobsthal and Pell Curves. The Fibonacci Quarterly 26.1 (1988), 79-83.

[14] K. V. Menon. On the convolution of logarithmically concave sequences, Proc. Amer. Math. Soc, 23 (1969), 439-441

[15] D. W. Walkup, Pólya sequences, binomial convolution and the union of random sets, J. Appl. Probab, 13 (1976), 76-85.

[16] M. Ahmia, H. Belbachir, Preserving log-concavity and generalized triangles. T. Komatsu (ed.), Diophantine analysis and related fields 2010. NY: American Institute of Physics (AIP). AIP Conference Proceedings 1264 (2010), 81-89.

[17] M. Ahmia, H. Belbachir, Preserving log-convexity for generalized Pascal triangles, Electron. J. Combin. 19(2) (2012), Paper 16, 6 pp.

[18] F. Brenti, Unimodal, log-concave and Pólya frequency sequences in combinatorics, Mem. Amer. Math. Soc. no. 413 (1989).

[19] H. Davenport, G. Pólya, On the product of two power series, Canadian J. Math. 1 (1949), 1-5.

[20] L. Liu, Y. Wang, On the log-convexity of combinatorial sequences, Advances in Applied Mathematics 39(4) (2007), 453-476. 